\title{
Synthesis and Antimicrobial Study of Thiophene Clubbed Thiazolyl Carbohydrazides
}

\author{
SADHANA DHONDIBHAU MHASKE \\ Department of Chemistry, Dadapatil Rajale Arts, Science and Commerce College, \\ Adinathnagar, 414505, (Affiliated to SPPU, Pune), Maharashtra, India. \\ *Corresponding author E-mail: mhaskesadhana@gmail.com \\ http://dx.doi.org/10.13005/ojc/370412
}

(Received: May 17, 2021; Accepted: August 18, 2021)

\begin{abstract}
Thiophene containing thiazolyl carbohydrazide on reaction with various aryl isothiocynates yields thiosemicarbazides which were transformed into 1,2,4-substituted thiazoles by Hantzsch synthesis and characterized by spectral methods. Most of the synthesized new thiosemicarbazides are found to be promisingly effective against tested bacterial strains and exhibited moderate activity tested fungal strains. Most of the 1, 2, 4-substituted thiazoles are weakly active against test organisms.
\end{abstract}

Keywords: Acid hydrazide, Thiosemicarbazides, Thiazole, Antimicrobial activity.

\section{INTRODUCTION}

Heterocyclic compounds containing sulphur ${ }^{1}$ are important entities present in various bioactive molecules in the field of medicinal and synthetic organic chemistry. Among the five membered ring containing sulphur heterocycles, thiazole ${ }^{2-5}$ and thiophene $e^{6-8}$ possess promising bioactivity profile. Compounds containing thiophene and thiazole ${ }^{9-14}$ moieties exhibited promising bioactivities like antimicrobial and antitumor activities.

Thiosemicarbazide and substituted thiosemicarbazides have been proved not only as efficient precursors for different heterocycles ${ }^{15}$ but also as potentially bioactive scaffolds ${ }^{16,-18}$. Synthesis of thiosemicarbazides from acid hydrazide derivatives ${ }^{18,19}$ is one of the effective synthetic routes. Acid hydrazides can be synthesized from esters ${ }^{20,21}$. Although thiazole and substituted thiazoles can be synthesized by various routes ${ }^{22-25}$, Hantzsch synthesis ${ }^{26-28}$ is an efficient and widely preferred route of thiazole synthesis. Phenacyl bromides are widely used in thiazole synthesis ${ }^{29}$. In our previous work we have reported antibacterial activities of bromine containing compounds ${ }^{30}$.

Activities associated with thiophene, thiazoles, thienyl-thiazoles, bromine containing compounds, synthetic and biological importance of thiosemicarbazides, phenacyl bromides and efficiency of synthetic routes prompted to club thiophene and thiazoles into carbohydrazides and evaluating them for antimicrobial potential.

This is an Open Access article licensed under a Creative Commons license: Attribution 4.0 International (CC- BY). Published by Oriental Scientific Publishing Company @ 2018

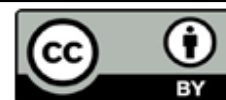




\section{RESULTS AND DISCUSSION}

Preparation of 4-Methyl-2-(thiophen-2-yl)1,3-thiazole-5-carbohydrazide 4 was carried out by known method ${ }^{20,21}$ (Scheme 1). The reaction of compound 4 and aryl isothiocynates 5 a-j in ethanol resulted in substituted thiosemicarbazides $6 \mathrm{a}-\mathrm{j}$ as shown in Scheme 2. The band at $1660 \mathrm{~cm}^{-1}$ in the IR spectrum of $6 \mathrm{a}$ was due to $\mathrm{C}=\mathrm{O}$ stretching. In the ${ }^{1} \mathrm{H}$ NMR spectrum of $6 \mathrm{a}$ a singlet at $\delta 2.69$ supports the presence of methyl group on thiazole ring, a triplet at $\delta 7.05$ with $\mathrm{J}=8.68 \mathrm{~Hz}$ for two protons is for two protons ortho to fluorine. The presence of $\mathrm{NH}$ protons was confirmed by singlets at $\delta 9.71,9.74$ for two protons and at $\delta 10.18$ for one proton. Thiosemicarbazides on condensation with 4-bromo-phenacyl bromide in ethanol were transformed into thiazoles 7a-j (Scheme 2). The bands at 3396 and $1666 \mathrm{~cm}^{-1}$ corresponding to $\mathrm{N}-\mathrm{H}$ stretching and for highly conjugated $\mathrm{C}=\mathrm{O}$ group respectively are seen in the IR spectrum of compound $7 a$. A singlet $\delta 2.71$ was for a deshielded methyl group on thiazole ring, three multiplets at $\delta$ 7.14-7.33, 7.43-7.45 and 7.60-7.79 for aromatic protons and a downfield singlet at $\delta 10.55$ for $\mathrm{N}-\mathrm{H}$ proton are seen in the ${ }^{1} \mathrm{H}$ NMR spectrum of the compound showed. Mass spectrometric analyses were in support of these compounds.

\section{Antimicrobial activity}

In vitro antimicrobial studies of the compounds $6 \mathrm{a}-\mathrm{j}, 7 \mathrm{a}$-j was determined against three bacterial strains Escherichia coli, Salmonella typhii and Bacillus subtilis and two fungal strains Alternaria and Fusarium oxysorum (Table 1). For this agar well diffusion method was used. Ciprofloxacin and ketoconazole were used as reference antibacterial and antifungal agents while DMSO is used as negative control. The results were recorded as an average of three experimental sets and expressed as zone of inhibition in $\mathrm{mm}$.

The results of antimicrobial study depicted that compounds $6 \mathrm{a}$-j are promisingly active against three bacterial strains while $6 \mathrm{a}-\mathrm{f}$ are active against Alternaria and 6b, $6 \mathrm{i}$ have activity against F. oxysporum. Among the remaining compounds $7 \mathrm{i}$, $7 \mathrm{~h}-\mathrm{j}$ are active against Bacillus subtilis. Compounds 7c, $7 \mathrm{~g}$ showed activity against Salmonella typhi. Compounds $7 \mathrm{i}, 7 \mathrm{c}$ exhibited fairly good activity against $F$. oxysporum. Rest of the compounds are weakly active or inactive at the $1 \mathrm{mg} / \mathrm{mL}$ concentration against experimental microbes. All the thiosemicarbazides showed promising antimicrobial activities while on cyclization activities have been diminished. The observed activities are may be due to presence of free $\mathrm{C}=\mathrm{S}$ group in compounds $6 \mathrm{a}-\mathrm{f}$.

Table 1: Antimicrobial Activity (Zone of Inhibition at $1 \mathrm{mg} / \mathrm{mL}$ in $\mathrm{mm}$ )

\begin{tabular}{|c|c|c|c|c|c|}
\hline Compound & Bacillus subtilis & $\begin{array}{c}\text { Antibacterial activity } \\
\text { Escherichia coli }\end{array}$ & Salmonella typhii & $\begin{array}{c}\text { Antifungal activity } \\
\text { Alternaria }\end{array}$ & Fusarium oxysporum \\
\hline $6 b$ & 13 & 12 & 12 & 21 & 15 \\
\hline $6 c$ & 20 & 16 & 18 & 26 & + \\
\hline $6 d$ & 18 & 15 & 17 & 19 & + \\
\hline $6 f$ & 13 & 15 & 21 & 18 & + \\
\hline $6 g$ & 19 & 17 & 17 & - & + \\
\hline $6 \mathrm{~h}$ & 15 & 14 & 13 & + & + \\
\hline $6 i$ & 13 & 13 & 15 & 20 & 16 \\
\hline $6 \mathrm{j}$ & 11 & 12 & 15 & + & + \\
\hline $7 e$ & - & - & - & - & + \\
\hline $7 f$ & - & - & - & - & + \\
\hline $7 g$ & + & - & 14 & + & - \\
\hline $7 \mathrm{~h}$ & 17 & - & + & + & + \\
\hline $7 i$ & 15 & - & - & - & \\
\hline $7 \mathrm{j}$ & 15 & - & + & + & + \\
\hline Ciprofloxacin & 35 & 40 & 39 & - & - \\
\hline Ketoconazole & - & - & - & 34 & 38 \\
\hline
\end{tabular}




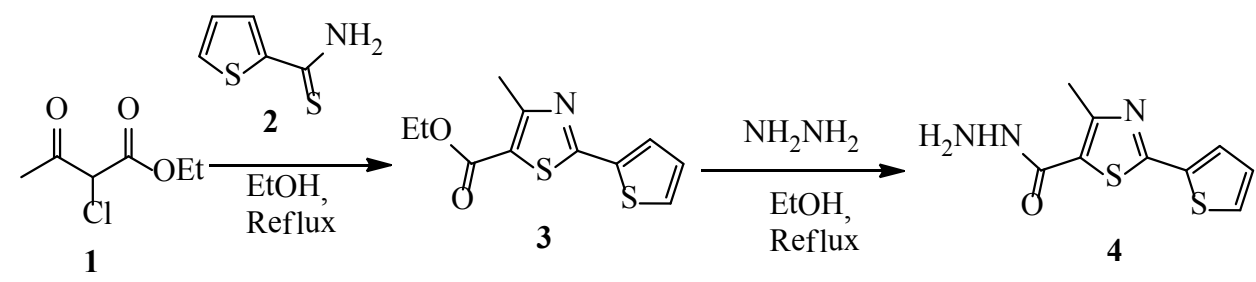

Scheme 1<smiles>Cc1nc(-c2cccs2)sc1C(=O)NN</smiles>

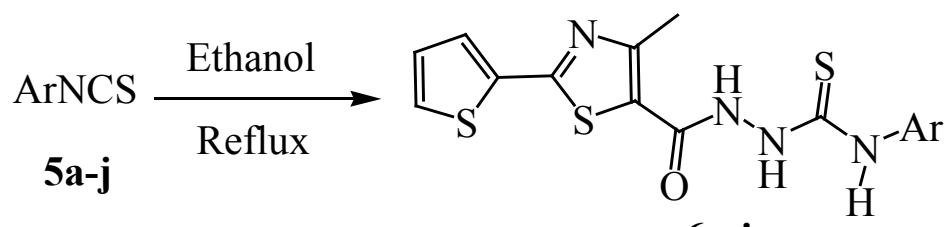

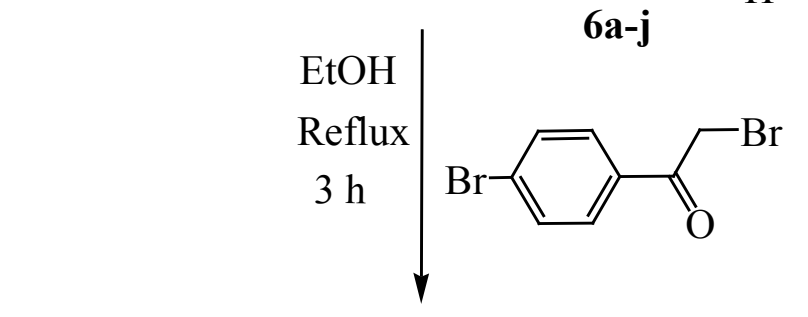

\section{EXPERIMENTAL}

Physical constants were recorded using open glass capillary method. The IR and NMR spectra were recorded using IRAffinity-1S spectrophotometer (Shimadzu) and Bruker Avance II $400 \mathrm{MHz}$ NMR spectrometer respectively. DMSO- $d_{6}$ was used as a solvent and TMS as reference compound in NMR experiment on HP 1100 LC/MSD Mass Spectrometer and Perkin-Elmer analyzer were used for mass spectrometric analyses and elemental analyses respectively.

$\mathrm{N}$-(Aryl)-2-\{[4-methyl-2-(thiophen-2-yl)-1,3thiazol-5-yl]carbonyl\}hydrazinecarbothioamides, 6a-j

Compound 4 and aryl isothiocynates $5 \mathrm{a}$ in equimolar quantity were refluxed for $1 \mathrm{~h}$ in $25 \mathrm{~mL}$ ethanol with TLC monitoring. The reaction mixture was left undisturbed for $15 \mathrm{~min}$ after completion of reaction. The solid product $6 a$ was filtered and recrystallized from ethanol. Preparation of compounds $6 b-j$ was<smiles>Cc1nc(C2=CCSS2)sc1C(=O)N/N=c1\scc(-c2ccc(Br)cc2)n1[Al]</smiles>

Scheme 2

achieved under same experimental condition. Physical data of compounds $6 \mathrm{a}-\mathrm{j}$ is mentioned in Table 2 and analytical data is given below.

Table 2: Physical data of synthesized compounds

\begin{tabular}{|c|c|c|c|}
\hline Compound & $\mathrm{Ar}$ & m.p. $\left({ }^{\circ} \mathrm{C}\right)$ & Yield(\%) \\
\hline $6 a$ & $4-\mathrm{F}-\mathrm{C}_{6} \mathrm{H}_{4}$ & 188 & 73 \\
\hline $6 b$ & $4-\mathrm{CH}_{3}-\mathrm{C}_{6} \mathrm{H}_{4}$ & 212 & 72 \\
\hline $6 c$ & $2-\mathrm{Cl}^{3} \mathrm{C}_{6} \mathrm{H}_{4}^{4}$ & 272 & 75 \\
\hline $6 d$ & $3-\mathrm{Cl}-\mathrm{C}_{6}^{6} \mathrm{H}_{4}^{4}$ & 242 & 75 \\
\hline $6 e$ & $4-\mathrm{Cl}-\mathrm{C}_{6}^{6} \mathrm{H}_{4}^{4}$ & 194 & 78 \\
\hline $6 f$ & 2,4-di-Cl- $\mathrm{C}_{6} \mathrm{H}_{3}$ & 291 & 74 \\
\hline $6 g$ & 3,4-di-Cl- $\mathrm{C}_{6} \mathrm{H}_{3}$ & 280 & 77 \\
\hline $6 h$ & $2-\mathrm{F}-\mathrm{C}_{6} \mathrm{H}_{4}$ & 172 & 71 \\
\hline $6 i$ & $3-\mathrm{CH}_{3}-\mathrm{C}_{6} \mathrm{H}_{4}$ & 222 & 73 \\
\hline $6 \mathrm{j}$ & $2-\mathrm{OCH}_{3}-\mathrm{C}_{6} \mathrm{H}_{4}$ & 182 & 72 \\
\hline $7 a$ & $4-\mathrm{F}-\mathrm{C}_{6} \mathrm{H}_{4}$ & 180 & 66 \\
\hline $7 b$ & 4- $\mathrm{CH}_{3}-\mathrm{C}_{6} \mathrm{H}_{4}$ & 174 & 67 \\
\hline $7 c$ & $2-\mathrm{Cl}-\mathrm{C}_{6} \mathrm{H}_{4}$ & 198 & 65 \\
\hline $7 d$ & $3-\mathrm{Cl}-\mathrm{C}_{6} \mathrm{H}_{4}$ & 204 & 60 \\
\hline $7 e$ & $4-\mathrm{Cl}-\mathrm{C}_{6} \mathrm{H}_{4}$ & 220 & 62 \\
\hline $7 f$ & 2,4-di-Cl- $\mathrm{C}_{6} \mathrm{H}_{3}$ & 178 & 64 \\
\hline $7 g$ & 3,4-di-Cl- $\mathrm{C}_{6} \mathrm{H}_{3}$ & 158 & 67 \\
\hline $7 \mathrm{~h}$ & $2-\mathrm{F}-\mathrm{C}_{6} \mathrm{H}_{4}$ & 162 & 65 \\
\hline $7 i$ & $3-\mathrm{CH}_{3}-\mathrm{C}_{6} \mathrm{H}_{4}$ & 168 & 68 \\
\hline $7 \mathrm{j}$ & $2-\mathrm{OCH}_{3}-\mathrm{C}_{6} \mathrm{H}_{4}$ & 260 & 63 \\
\hline
\end{tabular}


6a. IR: $3307,3169(\mathrm{~N}-\mathrm{H}$ stretching frequency), 1660 ( $\mathrm{C}=\mathrm{O}$ stretching frequency), 1608 ( $\mathrm{C}=\mathrm{N}$ stretching frequency), 1543 ( $\mathrm{Ar} \mathrm{C}=\mathrm{C}$ stretching frequency), 1213 (Ar-F stretching frequency), 827, $734 \mathrm{~cm}^{-1}$ (=C-H bending); ${ }^{1} \mathrm{H}$ NMR: $\delta 2.69(3 \mathrm{H}, \mathrm{s})$, $7.05(2 \mathrm{H}, \mathrm{t}, \mathrm{J}=8.68 \mathrm{~Hz}), 7.13-7.17(1 \mathrm{H}, \mathrm{m}), 7.36-$ $7.58(2 \mathrm{H}, \mathrm{m}), 7.60-7.62(2 \mathrm{H}, \mathrm{m}), 9.71(1 \mathrm{H}, \mathrm{s}), 9.74$ $(1 \mathrm{H}, \mathrm{s}), 10.18(1 \mathrm{H}, \mathrm{s})$; MS: $(\mathrm{M}+1) 393$; Analysis: $\mathrm{C}_{16} \mathrm{H}_{13} \mathrm{FN}_{4} \mathrm{OS}_{3}$ : Cal.: C, 48.96; H, 3.34; N, 14.27; Observed: C, 48.97; H, 3.33; N, 14.24\%.

6b. IR: $3257,3151(\mathrm{~N}-\mathrm{H}$ stretching frequency), 1654 ( $\mathrm{C}=\mathrm{O}$ stretching frequency), 1598 ( $\mathrm{C}=\mathrm{N}$ stretching frequency), $867,739 \mathrm{~cm}^{-1}(=\mathrm{C}-\mathrm{H}$ bending); ${ }^{1} \mathrm{H}$ NMR: $\delta 2.31(3 \mathrm{H}, \mathrm{s}), 2.68(3 \mathrm{H}, \mathrm{s})$, 7.12-7.18 $(3 \mathrm{H}, \mathrm{m}), 7.38-7.48(4 \mathrm{H}, \mathrm{m}), 7.55(1 \mathrm{H}, \mathrm{s})$, $9.48(1 \mathrm{H}, \mathrm{s}), 9.59(1 \mathrm{H}, \mathrm{s}), 10.02(1 \mathrm{H}, \mathrm{s})$; MS: $(\mathrm{M}+1)$ 389;Analysis: $\mathrm{C}_{17} \mathrm{H}_{16} \mathrm{~N}_{4} \mathrm{OS}_{3}$ : Cal.: C, 52.55; $\mathrm{H}, 4.15$; N, 14.42; Observed: C, 52.54; H, 4.12; N, 14.39\%.

6c. IR: $3317,3163(\mathrm{~N}-\mathrm{H}$ stretching frequency), 1651 ( $\mathrm{C}=\mathrm{O}$ frequency), 1610 ( $\mathrm{C}=\mathrm{C}$ frequency), $835,725 \mathrm{~cm}^{-1}$ (=C-H bending); ${ }^{1} \mathrm{H}$ NMR: ठ $2.69(3 \mathrm{H}, \mathrm{s}), 2.77(3 \mathrm{H}, \mathrm{s}), 7.15-7.25(4 \mathrm{H}, \mathrm{m})$, 7.35-7.50 (3H, m), $9.51(1 \mathrm{H}, \mathrm{s}) 9.62(1 \mathrm{H}, \mathrm{s}), 10.09$ (1H, s); MS: $(\mathrm{M}+1)$ 409; Analysis: $\mathrm{C}_{16} \mathrm{H}_{13} \mathrm{CIN}_{4} \mathrm{OS}_{3}$ : Cal.: C, 46.99; H, 3.20; N, 13.70; Observed: C, $46.96 ; \mathrm{H}, 3.17 ; \mathrm{N}, 13.68 \%$.

6d. IR: $3319,3225(\mathrm{~N}-\mathrm{H}$ stretching frequency), 1651 ( $\mathrm{C}=\mathrm{O}$ frequency), 1596 ( $\mathrm{C}=\mathrm{C}$ frequency), 834, $743 \mathrm{~cm}^{-1}$ (=C-H bending); ${ }^{1} \mathrm{H}$ NMR: $2.71(3 \mathrm{H}, \mathrm{s}), 7.13-7.20(4 \mathrm{H}, \mathrm{m}), 7.32-7.51(3 \mathrm{H}, \mathrm{m})$, $9.47(1 \mathrm{H}, \mathrm{s}), 9.58(1 \mathrm{H}, \mathrm{s}), 10.04(1 \mathrm{H}, \mathrm{s})$; $\mathrm{MS}:(\mathrm{M}+1)$ 409; Analysis: $\mathrm{C}_{16} \mathrm{H}_{13} \mathrm{CIN}_{4} \mathrm{OS}_{3}$ : Cal.: C, 46.99; $\mathrm{H}, 3.20$; N, 13.70; Observed: C, 46.98; H, 3.18; N, 13.69\%.

6e. IR: $3301,3192(\mathrm{~N}-\mathrm{H}$ stretching frequency), 1652 ( $\mathrm{C}=\mathrm{O}$ stretching frequency), 1547 ( $\mathrm{Ar} \mathrm{C}=\mathrm{C}$ stretching frequency), $837 \mathrm{~cm}^{-1} ;{ }^{1} \mathrm{H}$ NMR: ठ $2.72(3 \mathrm{H}, \mathrm{s}), 7.02(2 \mathrm{H}, \mathrm{d}, \mathrm{J}=8.2 \mathrm{~Hz}), 7.14-7.21$ $(3 \mathrm{H}, \mathrm{m}), 7.40-7.47(2 \mathrm{H}, \mathrm{m}), 9.49(1 \mathrm{H}, \mathrm{s}), 9.54$ (2H, bs), 10.12 (1H, s); MS: (M+1) 409; Elemental analysis: $\mathrm{C}_{16} \mathrm{H}_{13} \mathrm{ClN}_{4} \mathrm{OS}_{3}$ : Cal.: C, 46.99; $\mathrm{H}, 3.20$; N, 13.70; Observed: C, 46.96; H, 3.18; N, 13.69\%.

6f. IR: 3316, 3192 (N-H stretching frequency), 1652 ( $\mathrm{C}=\mathrm{O}$ stretching frequency), 1546 (Ar $\mathrm{C}=\mathrm{C}$ stretching frequency), $837 \mathrm{~cm}^{-1}(=\mathrm{C}-\mathrm{H}$ bending); $1 \mathrm{H} \mathrm{NMR:} \delta 2.68(3 \mathrm{H}, \mathrm{s}), 7.09-7.20(4 \mathrm{H}, \mathrm{m})$, 7.36-7.47 (2H, m), $9.48(1 \mathrm{H}, \mathrm{s}), 9.57(1 \mathrm{H}, \mathrm{s}), 10.02$
(1H, s); MS: $(\mathrm{M}+1)$ 443. Analysis: $\mathrm{C}_{16} \mathrm{H}_{12} \mathrm{Cl}_{2} \mathrm{~N}_{4} \mathrm{OS}_{3}$ : Cal. C, 43.34; H, 2.73; N, 12.64; Observed: C, 43.32; $\mathrm{H}, 2.71 ; \mathrm{N}, 12.63$.

6g. IR: $3312,3192(\mathrm{~N}-\mathrm{H}$ stretching frequency), 1654 ( $\mathrm{C}=\mathrm{O}$ stretching frequency), 1546 (Ar $\mathrm{C}=\mathrm{C}$ stretching frequency), $835 \mathrm{~cm}^{-1}(=\mathrm{C}-\mathrm{H}$ bending); ${ }^{1} \mathrm{H} \mathrm{NMR}: \delta 2.71(3 \mathrm{H}, \mathrm{s}), 6.84-6.9(2 \mathrm{H}, \mathrm{m})$, 7.06-7.21 $(4 \mathrm{H}, \mathrm{m}), 7.38-7.51(2 \mathrm{H}, \mathrm{m}), 9.50(1 \mathrm{H}, \mathrm{s})$, $9.59(1 \mathrm{H}, \mathrm{s}), 10.11(1 \mathrm{H}, \mathrm{s})$; MS: $(\mathrm{M}+1) 443$; Analysis: $\mathrm{C}_{16} \mathrm{H}_{12} \mathrm{Cl}_{2} \mathrm{~N}_{4} \mathrm{OS}_{3}$ : Cal.: $\mathrm{C}, 43.34 ; \mathrm{H}, 2.73 ; \mathrm{N}, 12.64$; Observed: C, 43.33; H, 2.72; N, 12.63\%.

6h. IR: $3304,3193(\mathrm{~N}-\mathrm{H}$ stretching frequency), 1651 ( $\mathrm{C}=\mathrm{O}$ stretching frequency), 1555 (Ar $\mathrm{C}=\mathrm{C}$ stretching frequency), $831 \mathrm{~cm}^{-1} ;{ }^{1} \mathrm{H}$ NMR: $\delta$ $2.76(3 \mathrm{H}, \mathrm{s}), 7.06-7.20(4 \mathrm{H}, \mathrm{m}), 7.36-7.45(3 \mathrm{H}, \mathrm{m})$, $9.51(1 \mathrm{H}, \mathrm{s}), 9.59(1 \mathrm{H}, \mathrm{s}), 10.15(1 \mathrm{H}, \mathrm{s})$; $\mathrm{MS}:(\mathrm{M}+1)$ 393; Analysis: $\mathrm{C}_{16} \mathrm{H}_{13} \mathrm{FN}_{4} \mathrm{OS}_{3}$ : Cal.: $\mathrm{C}, 48.96$; $\mathrm{H}, 3.34$; N, 14.27. Observed: C, 48.98; H, 3.32; N, $14.25 \%$.

6i. IR: $3307,3169(\mathrm{~N}-\mathrm{H}$ stretching frequency), 1660 ( $\mathrm{C}=\mathrm{O}$ stretching frequency), 1608 ( $\mathrm{C}=\mathrm{N}$ stretching frequency), 1504 ( $\mathrm{Ar} \mathrm{C}=\mathrm{C}$ stretching frequency), 1213, 827, $707 \mathrm{~cm}^{-1}$ (=C-H bending); ${ }^{1} \mathrm{H}$ NMR: $\delta 2.34(3 \mathrm{H}, \mathrm{s}), 2.72(3 \mathrm{H}, \mathrm{s}), 6.96-9.98$ $(1 \mathrm{H}, \mathrm{m}), 7.15-7.17(1 \mathrm{H}, \mathrm{m}), 7.19-7.36(3 \mathrm{H}, \mathrm{m}), 7.39-$ $7.42(2 \mathrm{H}, \mathrm{m}), 9.65(1 \mathrm{H}, \mathrm{s}), 9.68(1 \mathrm{H}, \mathrm{s}), 10.12(1 \mathrm{H}$, s); MS: $(M+1) 389$; Analysis: $\mathrm{C}_{17} \mathrm{H}_{16} \mathrm{~N}_{4} \mathrm{OS}_{3}$ : Cal.: $\mathrm{C}$, 52.55; H, 4.15; N, 14.42; Observed: C, 52.53; H, $4.11 ; \mathrm{N}, 14.38 \%$.

6j. IR: 3321,3192 (N-H stretching frequency), 1648 ( $\mathrm{C}=\mathrm{O}$ stretching frequency), 1549 (Ar $\mathrm{C}=\mathrm{C}$ stretching frequency), $827 \mathrm{~cm}-1 ; 1 \mathrm{H}$ NMR: $\delta 2.76$ $(3 \mathrm{H}, \mathrm{s}), 3.8(3 \mathrm{H}, \mathrm{s}), 6.84-6.9(2 \mathrm{H}, \mathrm{m}), 7.16-7.21(2 \mathrm{H}$, $\mathrm{m}), 7.37(2 \mathrm{H}, \mathrm{d}, \mathrm{J}=7.8 \mathrm{~Hz}), 7.95-8.05(2 \mathrm{H}, \mathrm{m}), 9.53$ (2H, bs), $10.01(1 \mathrm{H}, \mathrm{s})$; MS: $(\mathrm{M}+1)$ 404; Analysis: C17H16N4O2S3: Cal.: C, 50.47; H, 3.99; N, 13; Observed: C, 50.45; H, 3.97; N, 13.84\%.

\section{$N$-[(2Z)-4-(4-Bromophenyl)-3-phenyl-1,3-thiazol- 2(3H)-ylidene]-4-methyl-2-(thiophen-2-yl)-1,3- thiazole-5-carbohydrazides, 7a-j.}

In a $50 \mathrm{mLRBF}$, thiosemicarbazide $6 \mathrm{a}(0.001$ $\mathrm{mol})$ and 4-bromophenacylbromide $(0.001 \mathrm{~mol})$ were dissolved in $25 \mathrm{~mL}$ ethanol. A TLC monitored reaction completed in 3 hours. The reaction mixture was cooled to room temperature and crude compound 7 a was filtered, dried and purified by recrystallization from ethanol. Compounds $7 \mathrm{~b}$-j were prepared under 
similar experimental conditions. Physical data of compounds $7 \mathrm{a}-\mathrm{j}$ is mentioned in Table 2 and analytical data is given below.

7a. IR: 3396 (N-H stretching frequency), 1666 ( $\mathrm{C}=\mathrm{N}$ stretching frequency), 1604, 1573 ( $\mathrm{C}=\mathrm{C}$ stretching frequency), 1261 (Ar-F stretching frequency), 842, 812, $704 \mathrm{~cm}^{-1}$ (=C-H bending); ${ }^{1} \mathrm{H}$ NMR: $\delta 2.71(3 \mathrm{H}, \mathrm{s}), 3.74(3 \mathrm{H}, \mathrm{s}), 6.59(2 \mathrm{H}, \mathrm{s})$, 7.14-7.33 (7H, m), 7.43-7.45 (2H, m), 7.60-7.79 $(2 \mathrm{H}, \mathrm{m}), 10.55(1 \mathrm{H}, \mathrm{s})$; MS: $(\mathrm{M}+1) 571$; Analysis: $\mathrm{C}_{24} \mathrm{H}_{16} \mathrm{~N}_{4} \mathrm{~S}_{3} \mathrm{OBrF}: \mathrm{C}, 50.44 ; \mathrm{H}, 2.82 ; \mathrm{N}, 9.80$; Observed: C, 50.47; H, 2.84; N, 9.83\%.

7b. IR: 3361, 2921 (N-H stretching frequency), 1614,1570 ( $\mathrm{C}=\mathrm{C}$ stretching frequency), 841, 759, $701 \mathrm{~cm}^{-1}$ (=C-H bending); ${ }^{1} \mathrm{H}$ NMR: $\delta 2.31$ $(3 \mathrm{H}, \mathrm{s}), 2.75(3 \mathrm{H}, \mathrm{s}), 6.57(1 \mathrm{H}, \mathrm{s}), 7.08-7.18(4 \mathrm{H}, \mathrm{m})$, 7.20-7.36 (5H, m), $7.48(2 \mathrm{H}, \mathrm{d}, \mathrm{J}=8.5 \mathrm{~Hz}), 10.56$ $(1 \mathrm{H}, \mathrm{s})$; MS: $(\mathrm{M}+1)$ 567; Analysis: $\mathrm{C}_{25} \mathrm{H}_{19} \mathrm{~N}_{4} \mathrm{~S}_{3} \mathrm{OBr}$ : Cal.: C, 52.91; H, 3.37; N, 9.87; Observed: C, 52.92; $H, 3.38 ; N, 9.86 \%$.

7c. IR: 3351,2927 (N-H stretching frequency), 1626, 1581 ( $\mathrm{C}=\mathrm{C}$ stretching frequency), 835, 763, $704 \mathrm{~cm}^{-1}$ (=C-H bending); ${ }^{1} \mathrm{H}$ NMR: $\delta 2.70$ $(3 \mathrm{H}, \mathrm{s}), 6.58(1 \mathrm{H}, \mathrm{s}), 7.10(2 \mathrm{H}, \mathrm{d}, \mathrm{J}=8.2 \mathrm{~Hz}), 7.13-$ $7.35(7 \mathrm{H}, \mathrm{m}), 7.47(2 \mathrm{H}, \mathrm{d}, \mathrm{J}=8.2 \mathrm{~Hz}), 10.70(1 \mathrm{H}$, s); $M S:(M+1) 587$; Analysis: $\mathrm{C}_{24} \mathrm{H}_{16} \mathrm{~N}_{4} \mathrm{~S}_{3} \mathrm{OBrCl}$ : Cal.: C, 49.03; H, 2.74; N, 9.53; Observed: C, 49.06; H, $2.72 ; \mathrm{N}, 9.51 \%$.

7d. IR: 3361,2919 (N-H stretching frequency), 1622, 1582 ( $\mathrm{C}=\mathrm{C}$ stretching frequency), 845, 813, $702 \mathrm{~cm}^{-1}$ (=C-H bending); ${ }^{1} \mathrm{H}$ NMR: $\delta 2.69$ $(3 \mathrm{H}, \mathrm{s}), 6.57(1 \mathrm{H}, \mathrm{s}), 7.09-7.20(6 \mathrm{H}, \mathrm{m}), 7.26-7.38$ $(3 \mathrm{H}, \mathrm{m}), 7.46(2 \mathrm{H}, \mathrm{d}, \mathrm{J}=8.1 \mathrm{~Hz}), 10.61(1 \mathrm{H}, \mathrm{s})$; MS: (M+1) 587; Analysis: $\mathrm{C}_{24} \mathrm{H}_{16} \mathrm{~N}_{4} \mathrm{~S}_{3} \mathrm{OBrCl}$ : Cal.: C, 49.03; H, 2.74; N, 9.53; Observed: C, 49.04; H, 2.72; N, 9.55\%.

7e. IR: 3361, 2908 (N-H stretching frequency), 1619, 1581 ( $\mathrm{C}=\mathrm{C}$ stretching frequency), 843, 813, $702 \mathrm{~cm}^{-1}$ (=C-H bending); ${ }^{1} \mathrm{H}$ NMR $\left(\mathrm{DMSO}-d_{6}\right): \delta 2.71(3 \mathrm{H}, \mathrm{s}), 6.62(1 \mathrm{H}, \mathrm{s}), 7.06-$ $7.16(4 \mathrm{H}, \mathrm{m}), 7.19-7.35(5 \mathrm{H}, \mathrm{m}), 7.42(2 \mathrm{H}, \mathrm{d}$, $\mathrm{J}=8.4 \mathrm{~Hz}), 10.58(1 \mathrm{H}, \mathrm{s})$; MS: $(\mathrm{M}+1)$ 587; Analysis: $\mathrm{C}_{24} \mathrm{H}_{16} \mathrm{~N}_{4} \mathrm{~S}_{3} \mathrm{OBrCl}$ : Cal.: C, 49.03; H, 2.74; N, 9.53; Observed: C, 49.05; H, 2.76; N, 9.56\%. 7f. IR: 3352, 2933 ( $\mathrm{N}-\mathrm{H}$ stretching frequency), 1631, 1583 ( $\mathrm{C}=\mathrm{C}$ stretching frequency), 855, 803, $702 \mathrm{~cm}^{-1}$ (=C-H bending); ${ }^{1} \mathrm{H}$ NMR: $\delta 2.70$ $(3 \mathrm{H}, \mathrm{s}), 6.59(1 \mathrm{H}, \mathrm{s}), 7.05-7.20(5 \mathrm{H}, \mathrm{m}), 7.24-7.36$ $(3 \mathrm{H}, \mathrm{m}), 7.42(2 \mathrm{H}, \mathrm{d}, \mathrm{J}=8.2 \mathrm{~Hz}), 10.52(1 \mathrm{H}, \mathrm{s})$; MS: $(\mathrm{M}+1)$ 621; Analysis: $\mathrm{C}_{24} \mathrm{H}_{15} \mathrm{~N}_{4} \mathrm{~S}_{3} \mathrm{OBrCl}_{2}$ : Cal.: C, 46.31; H, 2.43; N, 9.00; Observed: C, 46.33; H, $2.45 ; \mathrm{N}, 9.03 \%$.

7g. IR: $3361,2934(\mathrm{~N}-\mathrm{H}$ stretching frequency), 1644, 1576 ( $\mathrm{C}=\mathrm{C}$ stretching frequency), 833, $707 \mathrm{~cm}^{-1}$; ${ }^{1} \mathrm{H}$ NMR: $\delta 2.72(3 \mathrm{H}, \mathrm{s}), 6.54$ $(1 \mathrm{H}, \mathrm{s}), 7.09(2 \mathrm{H}, \mathrm{d}, \mathrm{J}=8.4 \mathrm{~Hz}), 7.21-7.37(6 \mathrm{H}, \mathrm{m})$, $7.48(2 \mathrm{H}, \mathrm{d}, \mathrm{J}=8.4 \mathrm{~Hz}), 10.58(1 \mathrm{H}, \mathrm{s})$; MS: $(\mathrm{M}+1)$ 621; Analysis: $\mathrm{C}_{24} \mathrm{H}_{15} \mathrm{~N}_{4} \mathrm{~S}_{3} \mathrm{OBrCl}_{2}$ : Cal.: C, 46.31; $\mathrm{H}$, 2.43; N, 9.00; Observed: C, 46.30; H, 2.46; N, 9.01\%.

7h. IR: 3354, 2921 (N-H stretching frequency), 1623, 1573 ( $C=C$ stretching frequency), 842, 803, 700 $\mathrm{cm}^{-1}$ (=C-H bending); ${ }^{1} \mathrm{HNMR}: \delta 2.71(3 \mathrm{H}, \mathrm{s}), 6.56(1 \mathrm{H}$, s), 7.08-7.21 (5H, m), 7.24-7.38 $(4 \mathrm{H}, \mathrm{m}), 7.46(2 \mathrm{H}, \mathrm{d}$, $\mathrm{J}=8.4 \mathrm{~Hz}), 10.57(1 \mathrm{H}, \mathrm{s})$; MS: $(\mathrm{M}+1) 571$; Analysis: $\mathrm{C}_{24} \mathrm{H}_{16} \mathrm{~N}_{4} \mathrm{~S}_{3} \mathrm{OBrF}$ : Cal.: C, 50.44; H, 2.82; N, 9.80; Observed: C, 50.46; H, 2.84; N, 9.82\%.

7i. IR: 3351, 2919 ( N-H stretching frequency), 1603, 1572 ( $\mathrm{C}=\mathrm{C}$ stretching frequency), 845, 713, $702 \mathrm{~cm}^{-1}$ (=C-H bending); ${ }^{1} \mathrm{H}$ NMR: $\delta$ $2.32(3 \mathrm{H}, \mathrm{s}), 2.70(3 \mathrm{H}, \mathrm{s}), 6.56(1 \mathrm{H} \mathrm{s}),, 7.09(2 \mathrm{H}$, $\mathrm{d}, \mathrm{J}=8.4 \mathrm{~Hz}), 7.16-7.35(7 \mathrm{H}, \mathrm{m}), 7.49(2 \mathrm{H}, \mathrm{d}, \mathrm{J}=$ $8.4 \mathrm{~Hz}), 10.54(1 \mathrm{H}, \mathrm{s})$; MS: $(\mathrm{M}+1)$ 567; Analysis: $\mathrm{C}_{25} \mathrm{H}_{19} \mathrm{~N}_{4} \mathrm{~S}_{3} \mathrm{OBr}$ : Cal.: C, 52.91; $\mathrm{H}, 3.37 ; \mathrm{N}, 9.87$; Observed: C, 52.94; H, 3.39; N, 9.85\%.

7j. IR: 3381,2936 ( N-H stretching frequency), 1605,1575 ( $C=C$ stretching frequency), $815,723,703 \mathrm{~cm}^{-1}$ (=C-H bending); $\delta 2.72(3 \mathrm{H}, \mathrm{s})$, $3.81(3 \mathrm{H}, \mathrm{s}), 6.52(2 \mathrm{H}, \mathrm{s}), 6.98-6.16(4 \mathrm{H}, \mathrm{m}), 7.20$ $7.34(5 \mathrm{H}, \mathrm{m}), 7.44(2 \mathrm{H}, \mathrm{d}, \mathrm{J}=8.4 \mathrm{~Hz}), 10.57(1 \mathrm{H}$, s); MS: $(\mathrm{M}+1)$ 582; Analysis: $\mathrm{C}_{25} \mathrm{H}_{19} \mathrm{~N}_{4} \mathrm{~S}_{3} \mathrm{O}_{2} \mathrm{Br}$ : Cal.: C, 51.46; H, 3.28; N, 9.60; Observed: C, 51.48; H, $3.29 ; \mathrm{N}, 9.63 \%$.

\section{CONCLUSION}

In present study thiophene and thiazole containing carbohydrazides are synthesized quantitatively and spectroscopic data well support the proposed compounds. Among the $\mathrm{N}-($ Aryl) $-2-\{[4-$ 
methyl-2-(thiophen-2-yl)-1,3-thiazol-5-yl]carbonyl\} hydrazinecarbothioamide compounds, 6a-j showed promising bactericidal activity against $B$. subtilis, E. coli, S. typhi. Most of the compounds from 6a-j series are effective against fungal species Alternaria except $6 \mathrm{~g}$. Most of the compounds from this series are weakly active against Fusarium oxysporum. Compounds from 7a-j series are either inactive or showed less activity against all the test organisms. Overall majority of the compounds reported in the present work can be developed into more active agents by performing structural modifications.

\section{ACKNOWLEDGEMENT}

The author is thankful to Management and Principal of Dadapatil Rajale Arts, Science and Commerce College, Adinathnagar, and also thankful to Guide Hon. Dr. B. K. Karale for providing research facilities and valuable guidance. The author is grateful to the Director, SAIF, Punjab University, Chandigarh, for their support in the form of spectroscopic investigations.

\section{Conflict of Interest}

The author declares no conflict of interest.

\section{REFERENCES}

1. Pathania, S.; Narang, R. K.; Rawal, R. K. Eur. J. Med. Chem., 2019, 180, 486-508.

2. Mishra, I.; Mishra, R.; Mujwar, S.; Chandra, P.; Sachan, N. A. J. Heterocycl. Chem., 2020, DOI: 10.1002/jhet.3970.

3. Abdul, R.; Cihangir, T. Eur. J. Med. Chem., 2015, 97, 911-927.

4. Borcea, A-M.; Ionut, I.; Crisan, O.; Oniga, O. Molecules, 2021, 26, (624), http://doi.org/1 0.3390/molecules/26030624.

5. Gumus, M.; Yakan, M.; Koca, I. Future Med. Chem., 2019, 11(15), 1979-1998.

6. Singh, A.; Singh, G.; Bedi, P. M. J. Heterocycl. Chem., 2020, DOI: 10.1002/jhet.3990.

7. Pramodh, B.; Prathap, K. N. C.; Hema, N. K.; Warad, I.; Loknath, N. K. J. Mol. Struc., 2021, 1229, 129587.

8. Shah, R.; Verma, P. K. Chem. Cent. J., 2018, 12, 137, https://doi.org/10.1186/s13065-018-0511-5.

9. Mabkhot, Y. N.; Barkat, A..; Al-Majid, A. M.; Alshahrani, S.; Yousuf, S.; Choudhary, M. I. Chem. Cent. J., 2013, 7(112), https://doi. org/10.1186/1752-153X-7-112.

10. Fadda, A. A.; Tawfik, E. H.; Selim, Y. A. Polycycl. Aromatic Compds., 2018, DOI: 10.1080/10406638.2018.1555174.

11. Bandock, S.; Fadaly, W.; Metwally, M. A.; Eur. J. Med. Chem., 2010, 45(9), 3692-3701.

12. Radwan, A. S.; Khalid, M. A. A. J. Heterocycl. Chem., 2019, https://doi.org/10.1002/jhet.3493.

13. Moharab, R. M.; Khalil, E. M.; Mayhoub, A. E.; Amira, E. M. A. J. Heterocycl. Chem., 2019, https://doi.org/10.1002/jhet.3870.

14. Rizk, O. H.; Shaaban, O. G.; Wahab, A. E. A. The Open Med. Chem. Journal., 2017, 11, 38-53.

15. Acharya, P. T.; Bhavsar, Z. A.; Jethava, D. J.; Patel, D. B.; Patel, H. D.; J. Mol. Struc., 2021, 1226, Part A, 129268.
16. Aly, A. A.; Hassan, A. A.; El-Shaimaa, S. M. J. Heterocycl. Chem., 2018, 55, 2196-2223.

17. Patel, D. B.; Darji, D. G.; Patel, K. R.; Rajani, D. P.; Rajani, S. D.; Patel, H. D. J. Heterocycl. Chem., 2020, 57(3), 1183-1200.

18. Karale, B. K.; Takate, S. J.; Salve, S. P.; Zaware, B. H.; Jadhav, S. S. Indian J. Chem. 2014, 53B, 339-344.

19. Majumdar, P.; Pati, A.; Patra, M.; Behera, R. K.; Behera, A. K.; Chem. Rev., 2014, 114, 2942-2977.

20. El Rayes, S. M. Molecules., 2010, 15(5), 6759-6772.

21. Rollas, S.; Gulerman, N.; Erdeniz, H. I/ Farmaco., 2002, 57, 171-174.

22. Hussein, W.; Turan-Zitouni, G. MOJ Bioorg. Org. Chem., 2018, 2(2), 52-55.

23. Pathania, S., Rawal, R. K. Chem. Heterocycl. Compds., 2020, 56, 445-454.

24. Nayak, S.; Gaonkar, S. L. Mini Rev. Med. Chem., 2019, 19(3), https://doi.org/10.2174 /1389557518666180816112151.

25. Ali, S. H.; Sayed, A. R. Synth. Commun., 2021, 51(5), 670-700.

26. Yogi, P.; Ashid, M.; Hussain, N.; Khan, S.; Joshi, A. Asian J. Chem., 2016, 28(4), 927-932.

27. Takate, S. J.; Shinde, A.D.; Karale, B. K.; Akolkar, H.; Nawale, L.; Sarkar, D.; Mhaske, P. C. Bioorg. Med. Chem. Lett., 2019, 29, 1199-1202.

28. Karale. B. K.; Takate, S. J.; Salve, S. P.; Zaware, B. H.; Jadhav, S. S. Indian J. Chem., 2015, 54B, 798-804.

29. Aly, A. A.; El-Sheref, E. M.; Brown, A. B.; Brase, S.; Nieger, M. J. Sulfur Chem., 2019, 40, 641-647.

30. Takate S. J.; Salve, S. P.; Dare, S. B.; Karale, B. K.; Akolkar, H. N.; Falke, D. B., Ghungurde R. B.; Mhaske, S. D. Indian J. Heterocycl. Chem., 2020, 30, 525-530. 